\title{
Speed-dependent resolution analysis of ultrafast laser-scanning fluorescence microscopy
}

\author{
Antony C. S. Chan, Terence T. W. Wong, Kenneth K. Y. Wong, Edmund Y. Lam, and Kevin K. Tsia* \\ Department of Electrical and Electronic Engineering, The University of Hong Kong, Hong Kong, China \\ *Corresponding author: tsia@hku.hk
}

Received December 5, 2013; revised January 30, 2014; accepted February 1, 2014; posted February 3, 2014 (Doc. ID 201885); published March 12, 2014

\begin{abstract}
The image resolution of an aberration-corrected laser-scanning fluorescence microscopy (LSFM) system, like all other classical optical imaging modalities, is ultimately governed by diffraction limit and can be, in practice, influenced by the noise. However, consideration of only these two parameters is not adequate for LSFM with ultrafast laser-scanning, in which the dwell time of each resolvable image point becomes comparable with the fluorescence lifetime. In view of the continuing demand for faster LSFM, we here revisit the theoretical framework of LSFM and investigate the impact of the scanning speed on the resolution. In particular, we identify there are different speed regimes and excitation conditions in which the resolution is primarily limited by diffraction limit, fluorescence lifetime, or intrinsic noise. Our model also suggests that the speed of the current laser-scanning technologies is still at least an order of magnitude below the limit ( $\sim \mathrm{sub}-\mathrm{MHz}$ to $\mathrm{MHz}$ ), at which the diffraction-limited resolution can be preserved. We thus anticipate that the present study can provide new insight for practical designs and implementation of ultrafast LSFM, based on emerging laser-scanning techniques, e.g., ultrafast wavelength-swept sources, or optical time-stretch. (C) 2014 Optical Society of America
\end{abstract}

OCIS codes: (170.2520) Fluorescence microscopy; (170.7160) Ultrafast technology; (180.2520)

Fluorescence microscopy; (180.5810) Scanning microscopy.

http://dx.doi.org/10.1364/JOSAB.31.000755

\section{INTRODUCTION}

With the advent of myriad naturally occurring or synthetic fluorophores as contrast agents, fluorescence microscopy has proven an indispensable tool for visualizing biological specimens with impressive image contrast as well as high spatial resolution [1]. Further assisted by the leapfrog development in laser technology, laser-scanning fluorescence microscopy (LSFM) allows efficient fluorescence excitation (either onephoton or multiphoton excitation), and thus allows visualization of different biomolecular structures in cells and tissues with superior contrast.

However, it is not trivial for LSFM to achieve real-time fluorescence imaging with temporal resolution down to milliseconds or even less - a time scale within which plenty of intricate biological dynamics can take place, e.g., neuronal action potentials, cellular chemical waves, and drug transport [2-4]. Its imaging speed is typically limited to $\sim 1-10$ frames per second, based on the raster-scan approach [2]. The frame rate of LSFM is predominantly governed by the point-scanning speed of the scanning technique. Attempts have been made to speed up the acquisition process by utilizing various laserscanning mechanisms. Among all, mechanically scanning mirrors, known as galvanometric mirrors, are widespread in the commercial LSFM systems. However, because of the mechanical inertia in all the galvanometric mirrors, including microelectro-mechanical system scanners, it only can provide a one-dimensional (1D) scan rate up to $\sim 1 \mathrm{kHz}$. Modest improvement in scan speed can be achieved by operating the mirrors at their resonant frequencies-mostly up to $\sim 10 \mathrm{kHz}$ [5]. Recent advances using a rotating polygonal mirror can scale the speed up to $\sim 100 \mathrm{kHz}$ [6]. Another type of laser scanning element is the acousto-optic deflector (AOD), in which the laser steering is accomplished based on diffraction by the frequency-dependent acoustic wave [7]. AOD scanners eliminate the inherent limitations of galvanometric scanners, i.e., speed and mechanical stability, and so can provide a faster scan rate up to $\sim 10-100 \mathrm{kHz}$ []. An emerging high-speed laser-scanning technique is spectral encoding, which employs a wavelength-swept laser source and a diffraction grating. This approach was demonstrated for LSFM of nonbiological specimens with a scan rate of $40 \mathrm{kHz}$ [9]. Notably, the scan rate of the spectral-encoding-based laser scanning can be drastically scaled up to $\sim 100 \mathrm{MHz}$ by an optical time-stretch process-a technique which has also been applied for ultrafast spectroscopy [10] as well as nonfluorescent imaging [10-16].

On the one hand, the laser-scanning technologies continue to boost the scanning speed for achieving the ultimate, highspeed LSFM; on the other hand, they are intrinsically limited by the fluorescence decay lifetimes of the fluorophores. Depending on the types of fluorophores and the ambient conditions, the typical fluorescence lifetimes for bioimaging are in the order of nanosecond to microsecond [17]. At an ultrafast scanning speed, the dwell time of each resolvable image point, defined by point spread function (PSF), becomes comparable with the decay lifetime of the fluorophore. Fluorescence decay tail [Fig. 1(a)] thus has to be taken into account when evaluating the PSF, or the resolution of the LSFM system. This is in contrast to the conventional aberration-free LSFM system, in which the PSF is fundamentally governed by the diffraction limit [1, $\underline{18}-\underline{21}]$. 


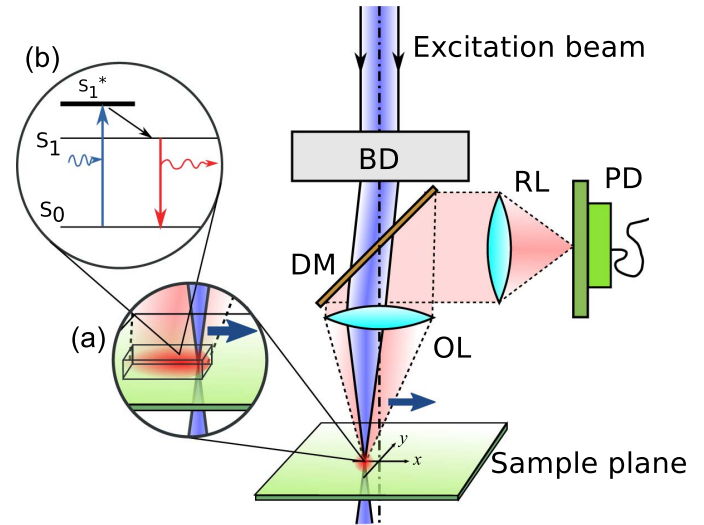

Fig. 1. Generic schematic of an LSFM system. (a) Zoom-in view of the smeared fluorescence emission due to the fluorescence lifetime decay tail, which becomes significant under the high-speed scanning operation. (b) Simplified Jablonski diagram showing the key energy states of the fluorophores. The energy transitions are also shown therein. (Key: BD: beam deflector; OL: objective lens; DM: dichroic mirror; RL: relay-lens system; PD: photodiode.)

To this end, here we present a theoretical analysis of the performance of LSFM under the high-speed laser-scanning scenario. We particularly revisit the resolution evaluation of LSFM by considering both the fluorescence dynamics and diffraction limit to investigate the impact of the scanning speed on the resolution. Such consideration has been overlooked in LSFM, as the fluorescence lifetime and resolution are mostly decoupled in the slow laser-scanning speed regime. Based on our model, we identify different speed regimes and excitation conditions, in which the resolution is primarily limited by (i) diffraction limit, (ii) fluorescence lifetime, and (iii) noise. In particular, we consider the intrinsic shot-noise of fluorescence emission in our model to investigate the effect of noise on the image under such high-speed operation. The analysis allows us to identify the practical speed limit of LSFM, below which the system can perform at reasonably high signal-tonoise (SNR). Our result also suggests that the speed of the current laser-scanning technologies is still at least an order of magnitude below the limit ( $\sim \mathrm{sub}-\mathrm{MHz}$ to $\mathrm{MHz}$ ) at which the diffraction-limited resolution can be preserved.

Extensive studies have been carried out to investigate the effect of noise on the fluorescence microscopy performance $[17,18,22-24]$. However, the knowledge of how the scanning speed, especially under ultrafast scanning, influences the noise of LSFM has been elusive. We anticipate that the present study could provide a new insight for design and implementation of ultrafast LSFM.

\section{GENERAL THEORETICAL FRAMEWORK}

\section{A. System Considerations}

A typical laser-scanning fluorescence microscope can be generalized by the schematic shown in Fig. 1. The laser-scanning fluorescence excitation is done by a beam deflector (BD) (e.g. galvanometric mirrors, AOD, or spectral-encoding scanner), which are located on the conjugate plane of the rear aperture of an objective lens (OL). The scanner provides angular deflection of the excitation beam. The OL, which is assumed to be telecentrically corrected, focuses the beam and transforms its angular steering motion to a linear scan motion across the specimen at the linear velocity $v^{*}$. The multiple-lens telecentric relay system, commonly equipped in the LSFM system, is omitted here for simplicity. A fraction of the fluorescence emission is collected back by the same lens (OL). A dichroic mirror (DM) is used to spectrally separate the emission light from the background excitation light, and redirects the emission light to a photodetector (PD) through another relay lens system $(\mathrm{RL})$. The $\mathrm{PD}$ is located on the conjugate plane of the specimen plane. In this paper, we primarily consider that neither de-scanning scheme nor confocal detection (i.e., no pinhole) is implemented. In this way, we can compare all the existing state-of-the-art laser-scanning technologies under the same detection conditions as beam descanning and, thus, confocal detection is nontrivial for some scanning mechanisms, including AOD and spectral-encoding [5,25]. Nevertheless, we note that our model is also applicable to the confocal detection with de-scanning as a special case.

\section{B. Theoretical Model}

\section{Fluorescence Excitation}

We here consider that a two-dimensional (2D) fluorescence image is captured by a raster-scan motion of a single laser beam. This model is applicable to all the major laser-scanning techniques (e.g., galvanometric mirrors, AOD, or spectralencoding scanner), including the multi-spot scanning approach [26], provided that the adjacent spots do not introduce cross talk of fluorescence signal. We primarily focus on the effect on the resolution of LSFM under ultrafast linear scanning motion (i.e., 1D across the specimen). Therefore, it suffices to consider the fluorophore distribution in the specimen to be $x$-dependent only and it is homogeneous in both the $y$ and $z$ directions.

We use the dimensionless time variable $t$ and space variable $x$, which represent the normalized time scale and length scale with respect to the lifetime $\tau$, and the excitation beam waist $w$, respectively. The actual length scale (indicating the beam displacement) $x^{*}$ and time scale $t^{*}$ are defined as $x^{*}=x w$ and $t^{*}=t \tau$.

We also define the "normalized" linear scanning speed $v$ of the excitation beam relative to its beam waist, within the fluorescence lifetime. The actual scanning speed $v^{*}$ is thus expressed as

$$
v^{*}=v \times w / \tau .
$$

The excitation beam is assumed to have a Gaussian profile and the excitation photon flux profile is

$$
\varphi_{\mathrm{ex}}(x ; t)=\frac{\bar{Q}_{\mathrm{ex}}}{2 \pi} \exp \left[-\frac{1}{2}(x-v t)^{2}\right],
$$

where $\bar{Q}_{\text {ex }}$ is the average photon rate from the illumination source per unit lifetime, defined as $\bar{Q}_{\mathrm{ex}}=\bar{P}_{\mathrm{ex}} \tau \lambda_{\mathrm{ex}} / h c$, where $\bar{P}_{\text {ex }}$ and $\lambda_{\text {ex }}$ are the average power and the wavelength of the excitation laser, respectively. $h$ is Planck's constant and $c$ is the speed of light in free space. The Gaussian excitation profile is chosen to establish an analytical model that provides a straightforward understanding of the speed-dependent performance of LSFM. The excitation spatial profile in Eq. (2) can be replaced by any other appropriate forms to represent different excitation configurations, such as an Airy disk function for an overfilled aberration-free OL [27]. Gaussian approximation is 
also well-known to be an accurate model to study the performance of fluorescence microscopy [27].

\section{Fluorescence Dynamics}

A vast majority of the previous theoretical work on LSFM investigated the low-scanning-speed LSFM systems (i.e., $v \ll 1$ ) [1,18-21], in which the fluorescence response is essentially in steady-state within the time scale required to traverse a focused beam waist (or simply a spot dwell time). However, at an ultrafast scanning speed $(v \geq 1)$, such a transit time is comparable with a unit of fluorescence lifetime. In other words, fluorescence afterglow may lead to signal cross talk between the neighboring spots. The transient response thus must be considered in the resolution analysis. The fluorescence dynamics is modeled here by a simplified two-level Jablonski diagram, as depicted in Fig. 1(b). Typically, the relaxation time within the same energy state $\left(S_{1}^{*} \rightarrow S_{1}\right)$ is negligibly short compared with the spot dwell time as well as the fluorescence lifetime ( 1-100 ns) [28]. Therefore, we assume this process to be instantaneous. We further assume the contributions from the inter-system crossing to the long-lived triplet states, as well as phosphorescence, to be negligible. This is particularly valid for the high-speed scanning scenario. We note that the fluorescence dynamics, and thus the lifetime, in practice, can be influenced by a number of factors, such as ambient $\mathrm{pH}$, temperature, quenching effect, dissociation of the fluorophores, and so on [1]. The fluorescence lifetime serves as the generic parameter linked to the radiative process, which ultimately influences image quality, as discussed in detail in the later section.

The total concentration of the fluorophores at any position $x$ and time $t, N_{\text {tot }}(x ; t)$, is then given by the sum of the fluorophore concentration in the ground states $N_{0}(x ; t)$ and that in the excited state $N_{1}(x ; t)$, i.e., $N_{\text {tot }}(x ; t)=N_{0}(x ; t)+$ $N_{1}(x ; t)$. We define the population probabilities of the ground state and the excited state as $p_{0}(x ; t)$ and $p_{1}(x ; t)$, such that $p_{0}(x ; t)+p_{1}(x ; t)=1$. In other words, the excited state concentration can be found as

$$
N_{1}(x ; t)=N_{\text {tot }}(x) p_{1}(x ; t)
$$

The fluorescence dynamics of the excited state is given by a rate equation in terms of $p_{1}(x ; t)$ :

$$
\frac{d p_{1}(x ; t)}{d t}=-p_{1}(x ; t)+\frac{a}{w^{2}} \varphi_{\mathrm{ex}}(x ; t) \times\left[1-p_{1}(x ; t)\right],
$$

where $a$ is the effective absorption cross section per fluorophore. Given a quantum efficiency $\eta_{q}$ of the radiative decay, the fluorescence emission flux is

$$
\varphi_{\mathrm{em}}(x ; t)=\eta_{q} N_{\mathrm{tot}}(x) p_{1}(x ; t) \Delta A,
$$

where $\Delta A$ is the effective emission cross section area in the $y-z$ plane, given by the specimen thickness and the beam size in the $y$-direction.

\section{Fluorescence Detection}

Let $h(x)$ be the PSF of the optical system, which includes the OL and other relay lenses before the PD. The detected fluorescence emission distribution in the $\mathrm{PD}$ plane, a conjugate plane of the specimen, is the convolution of the sample emission and the PSF $h(x)$ :

$$
\varphi_{\mathrm{em} 2}\left(x_{2} ; t\right)=\eta_{\mathrm{obj}}\left[\varphi_{\mathrm{em}}(x ; t) *|h(x)|^{2}\right]_{x=x_{2} / M},
$$

where $*$ is denoted as the convolution operator. $M$ is the magnification factor of the microscope system. The proportionality constant in Eq. (6) is the objective collection efficiency $\eta_{\mathrm{obj}}=\left(1-\sqrt{1-\mathrm{NA}^{2}}\right) / 2$, where NA is the numerical aperture of the OL [24]. Assuming an aperture size of $2 R$ (in a unit of $w$ ) at the PD, the overall emission photon flux $\bar{Q}_{\mathrm{em}}(t)$ is given by

$$
\begin{aligned}
Q_{\mathrm{em}}(t)= & \eta_{\mathrm{obj}} \eta_{q} \int_{-R}^{+R} \varphi_{\mathrm{em} 2}\left(x_{2} ; t\right) \mathrm{d} x_{2} w \Delta A \\
= & \eta_{\mathrm{obj}} \eta_{q} \int_{-\infty}^{+\infty} N_{\mathrm{tot}}(x) p_{1}(x ; t) \\
& \times \int_{-R / M}^{+R / M}\left|h\left(x^{\prime}-x\right)\right|^{2} \mathrm{~d} x^{\prime} \mathrm{d} x w \Delta A
\end{aligned}
$$

\section{Overall Model}

Combining Eqs. (2) and (4), we obtain a rate equation describing the spatiotemporal response of the excited fluorophores, with a scanning laser beam moving at a linear velocity $v$ :

$$
\frac{d p_{1}}{d t}=-p_{1}+\eta_{\mathrm{abs}} \bar{Q}_{\mathrm{ex}} \exp \left[-\frac{1}{2}(x-v t)^{2}\right] \times\left(1-p_{1}\right),
$$

where $\eta_{\mathrm{abs}}=a /\left(2 \pi w^{2}\right)$ is defined as photon absorption efficiency. If we assume that the fluorophores are far from saturation (i.e., $1-p_{1} \sim 1$ ), then Eq. (ㅇ) can be further reduced to:

$$
\frac{d p_{1}}{d t}=-p_{1}+\eta_{\mathrm{abs}} \bar{Q}_{\mathrm{ex}} \exp \left[-\frac{1}{2}(x-v t)^{2}\right],
$$

which can be solved analytically. As discussed in Section 2.A, a large-area detector is used (i.e., $R \gg w$ ); therefore, the detected emission photon flux in Eq. (7) is given by

$$
\bar{Q}_{\mathrm{em}}(t)=\eta_{\mathrm{obj}} \eta_{q} \int_{-\infty}^{+\infty} N_{\mathrm{tot}}(x) p_{1}(x ; t) \mathrm{d} x w \Delta A .
$$

We also define an overall photon transfer efficiency (PTE) $\gamma$, as the ratio of the average emission $\bar{Q}_{\mathrm{em}}$ to the average excitation photon flux $\bar{Q}_{\text {ex }}$. PTE is relevant to the noise analysis in later sections and can be derived from Eq. (10) in the nonsaturation condition

$$
\gamma=\bar{Q}_{\mathrm{em}} / \bar{Q}_{\mathrm{ex}}=\eta_{\mathrm{obj}} \eta_{q} \eta_{\mathrm{abs}} \times \bar{N}_{\mathrm{tot}} \times \sqrt{2 \pi} w \Delta A,
$$

where $\bar{N}_{\text {tot }}$ is the average concentration. See Appendix A for a detailed derivation.

\section{PERFORMANCE METRICS}

\section{A. Scanning-Speed-Dependent Resolution}

We here characterize the LSFM by the contrast transfer function (CTF), defined as the detected fluorescence signal contrast as a function of the spatial frequency $k$ of a fluorescence specimen. The contrast is defined as $C=\left(Q_{\mathrm{em}, \max }-\right.$ $\left.Q_{\mathrm{em}, \min }\right) /\left(2 \bar{Q}_{\mathrm{em}}\right)$, where $Q_{\mathrm{em}, \max }$ and $Q_{\mathrm{em}, \min }$ correspond to 


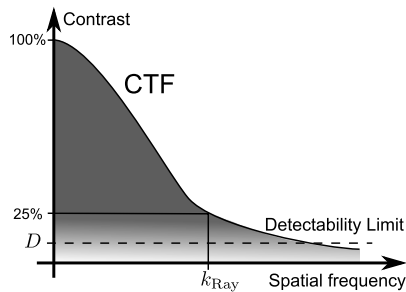

(a)

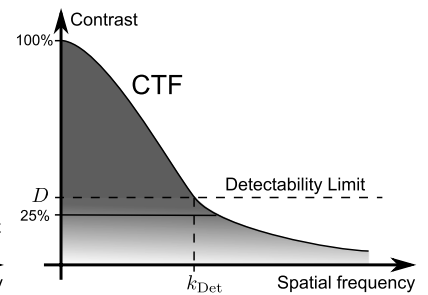

(b)
Fig. 2. Defining resolution by contrast transfer function (CTF). (a) Resolution determined by the cut-off spatial frequency $k_{\text {Ray }}$ at which the contrast $C=25 \%$ (i.e., Rayleigh criterion). This is the case when the detectability limit $D<25 \%$, i.e., a low-noise system whose resolution is either diffraction-limited or lifetime-limited. (b) Resolution defined by the cut-off spatial frequency $k_{\text {Det }}$ at which the contrast $C=D$. This corresponds to the case when $D>25 \%$, i.e., the noisy system whose resolution is noise-limited.

the detected maximum and minimum photon rate. $\bar{Q}_{\mathrm{em}}$ is the average photon rate. The resolution can be determined by the Rayleigh criterion-the smallest resolvable periodic feature giving a contrast of $25 \%$ [1]. In other words, it corresponds to a $25 \%$-bandwidth in a CTF with a cut-off spatial frequency $k_{\text {Ray }}$ [Fig. 2(a)].

Taking the scanning speed into account, we obtain the CTF of an LSFM system based on Eqs. (9) and (10) as (see Appendix A for detailed derivation):

$$
\mathrm{CTF}(k)=\frac{\exp \left(-k^{2} / 2\right)}{\sqrt{v^{2} k^{2}+1}},
$$

where $k$ is the normalized spatial frequency with a unit $2 \pi$. Equation (12) provides a simple but important concept that the scanning speed $v$ and, thus, the fluorescence lifetime $\tau$ [see Eq. (1)] come into play when evaluating the CTF and the resolution. This is clearly depicted in Fig. 3, showing the scanning-speed-dependent CTFs (solid lines). The contribution of fluorescence lifetime to CTF vanishes at very low scanning speed $(v \ll 1)$. In this case, CTF is reduced to the standard form, describing the classical LSFM:

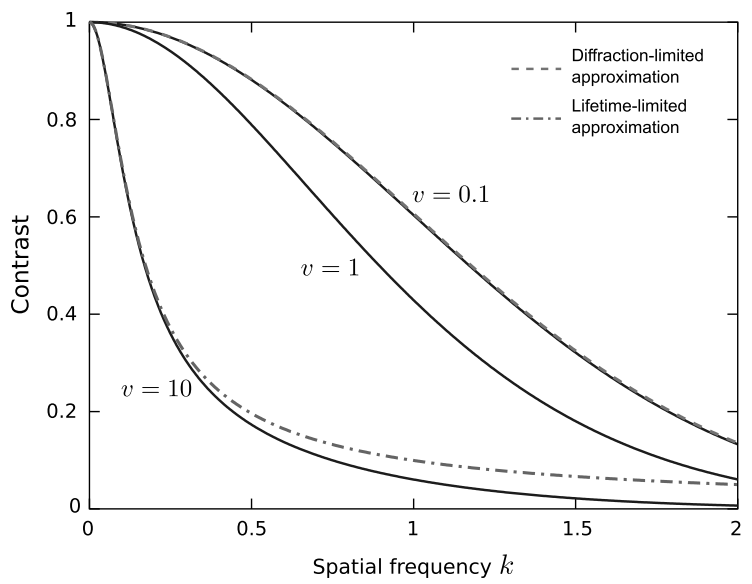

Fig. 3. Laser scanning-speed-dependent CTFs at the speed $v=0.1,1$, and 10. The dashed and dotted-dashed lines represent the diffractionlimited approximation [Eq. (13)] and the lifetime-limited approximation [Eq. (14)], respectively. As the speed increases, the bandwidth of the $\mathrm{CTF}$ becomes narrower, i.e., the contrast and the resolution are degraded correspondingly.

$$
\mathrm{CTF}(k) \simeq \exp \left(-0.5 k^{2}\right) \quad \text { if } v \ll 1 .
$$

This essentially corresponds to the diffraction-limited case (see the dotted line in Fig. 3 using this approximation). The resolution in this case can be obtained analytically by evaluating $k_{\text {Ray }}=\sqrt{\ln \left(0.25^{-2}\right)} \simeq 1.67$, which corresponds to a resolvable spatial period of $2 \pi w / k_{\text {Ray }} \simeq 2 \times 2 w$. In contrast, when speed $v$ is high $(v \gg 1)$, the contrast is progressively diminished, implying the deteriorating resolution. In this scenario, the CTF, and thus the resolution, are said to be lifetimelimited. The CTF in this limiting case can be approximated as:

$$
\mathrm{CTF}(k)=\frac{1}{\sqrt{v^{2} k^{2}+1}} \quad \text { if } v \gg 1 .
$$

Such lifetime-limited approximation is exemplified in Fig. 3 for $v=10$. The resolution bandwidth (25\%-contrast) $k_{\text {Ray }}=\sqrt{15 / v^{2}}$, and thus the resolvable period, is given as $2 \pi w / k_{\text {Ray }} \simeq 1.6 v w$. As a general case, the resolution (25\%contrast) bandwidth $k_{\text {Ray }}$ can be evaluated by

$$
\operatorname{CTF}\left(k_{\text {Ray }}\right)=\frac{\exp \left(-k_{\text {Ray }}^{2} / 2\right)}{\sqrt{v^{2} k_{\text {Ray }}^{2}+1}}=25 \%,
$$

and will be further discussed in Section 4.B.

\section{B. Noise-Limited Resolution}

In the previous section, the resolution is determined based on the assumption that the noise in the system is negligibly small. This means that the resolution is diffraction-limited at slow scan speeds $(v \ll 1)$, and lifetime-limited at fast scan speeds $(v>1)$. In reality, noise, a random fluctuation in detected fluorescence signals, could degrade the SNR as well as the contrast (Fig. 4). This is particularly relevant to the cases of ultrafast laser-scanning, i.e., shorter dwell time, resulting in fewer photons collected from fluorescence emission. To quantify the noise performance, we adopt the concept of detectability limit $D$ [29]:

$$
D=\frac{1}{\mathrm{SNR}}=\frac{\sigma}{m}
$$

where $\sigma$ is the variance in photon number, which is essentially related to the intensity noise, $m$ is the expected number of

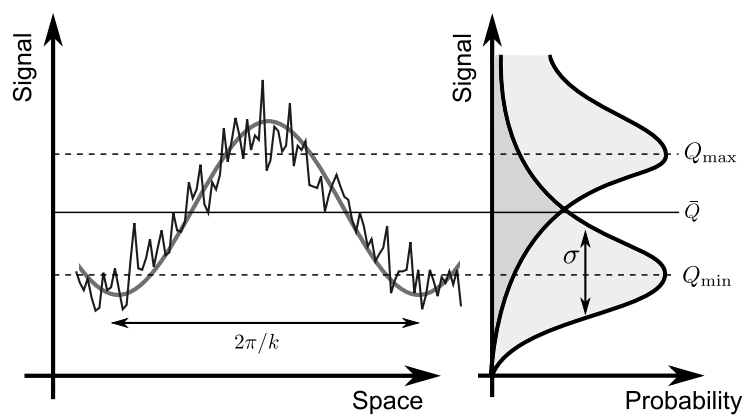

Fig. 4. Quantifying the impact of noise on image contrast. For a small object of size $2 \pi / k$ to be visible in the presence of noise fluctuation, signal range $\left(Q_{\max }-Q_{\min }\right)$ must be larger than signal variation $(\sigma)$. In the noise-limited case, $C=\left(Q_{\max }-Q_{\min }\right) /(2 \bar{Q})=\sigma / \bar{Q}=D$, where $D=1 /$ SNR is the detectability limit. 
photons received per image pixel, or equivalently the product of the emission photon rate, and the sampling interval $T$ of the PD:

$$
m=\bar{Q}_{\mathrm{em}} T=\gamma \bar{Q}_{\mathrm{ex}} T .
$$

In practice, the noise originates from the inherent shotnoise of the fluorescent photons, the dark current noise, and the thermal noise of the PD [1,22]. For the sake of argument, we assume ideal photodetection, in which the noise is only limited by the photon shot-noise, following the Poisson statistics [23]. It results in the shot-noise-limited detectability $D=1 / \sqrt{m}$. The detectability limit defines the smallest contrast that can be distinguished from the background (i.e., average signal) in the presence of noise. In other words, features having contrast smaller than the detectability limit will be indistinguishable. Therefore, we can also define $k_{\text {Det }}$ as the resolution bandwidth solely determined by detectability limit. The condition is given as

$$
\operatorname{CTF}\left(k_{\text {Det }}\right)=D=\frac{1}{\sqrt{m}}=\frac{1}{\sqrt{\gamma \bar{Q}_{\mathrm{ex}} T}} .
$$

For the system with low noise, the resolution is limited by the Rayleigh criterion as $D$ could be smaller than the $25 \%$ contrast level, i.e., $\operatorname{CTF}\left(k_{\text {Ray }}\right)>\operatorname{CTF}\left(k_{\text {Det }}\right)$ [Fig. 2(a)]. In contrast, for the system with low emission intensity or high-noise, the signal fluctuation would overwhelm the 25\%-contrast level, i.e., $\operatorname{CTF}\left(k_{\text {Ray }}\right)<\operatorname{CTF}\left(k_{\text {Det }}\right)$ [Fig. 2(b)] [1] $]$. In this case, the contrast should primarily be governed by the detectability limit. The resolution is thus said to be noise-limited.

It should be stressed that a proper sampling interval $T$ should be carefully chosen, especially in the ultrafast scanning regime. Intuitively, it is desirable to shorten $T$ at higher scan rates to avoid undersampling (i.e., pixelation in the image). However, smaller $T$ also implies loss of collected emission photons, and thus degrades the SNR. Yet, increasing $T$ can improve the SNR, but at the risk of undersampling, which results in loss of resolution. Therefore, the optimal $T$ should be chosen to satisfy the Nyquist sampling limit, which ensures to resolve the feature with a maximum cut-off spatial frequency $k_{\text {Det }}$ on the CTF, i.e., $T=0.5 \times 2 \pi /\left(v k_{\text {Det }}\right)$. Based on Eqs. (12) and (18), the value of $k_{\text {Det }}$ must satisfy the condition of

$$
\frac{\exp \left(-k_{\text {Det }}^{2} / 2\right)}{\sqrt{v^{2} k_{\text {Det }}^{2}+1}}=\left[\gamma \bar{Q}_{\mathrm{ex}} \times \frac{\pi}{v k_{\text {Det }}}\right]^{-1 / 2}
$$

Equation (19) is particularly useful for quantifying the noise-limited resolution, as discussed in Section 4.C.

\section{Effect of Fluorescence Saturation}

Equation (9) assumes that the excitation power does not deplete the ground state of the fluorophores. A good approximation for an unsaturated condition is that the excitation power is $10 \%$ of the saturation level [4]. This corresponds to

$$
\bar{Q}_{\mathrm{ex}}=\frac{0.1}{\eta_{\mathrm{abs}}}=0.1 \bar{Q}_{\mathrm{sat}},
$$

where $\bar{Q}_{\text {sat }}=1 / \eta_{\text {abs }}$ is the saturation photon rate [29]. In the case of saturation, the CTF is no longer a linear spaceinvariant function. Therefore, numerical simulation of Eqs. (8) and (10) is performed to investigate the saturation-dependent resolution. The results will be discussed in Sections $\underline{4 . \mathrm{B}}$ and 4.C.

\section{RESULTS AND DISCUSSION}

\section{A. Relationship between Spatial Resolution and Scanning Speed}

We first consider a noiseless LSFM system, i.e., based on Eq. (15), to analyze the key relationship between the spatial resolution and scanning speed, as shown in Fig. 5. Below the speed of $v \sim 0.3$, the resolution is essentially independent of the speed, while the resolution grows linearly with the scanning speed when it is beyond $v=0.3$. The significance of this plot becomes obvious if we compare the speeds of different state-of-the-art laser-scanning techniques (see also Table 1). Here, we assume full-sweep displacement or, equivalently, the field-of-view of $150 \mu \mathrm{m}$. This value corresponds to the case using a typical high-magnification OL (e.g. $>60 \times$ ). We found that the scanning speeds of most existing laser-scanning systems, including galvanometric mirrors $\left(v \sim 10^{-4}\right)$ and resonant mirrors $\left(v \sim 10^{-2}\right)$ are orders of magnitude slower than $v \simeq 1$. The resolutions achieved by these techniques are, thus, primarily diffraction-limited. It has been demonstrated that the fluorescence imaging speed can be boosted by employing an AOD scanner [ 8 ] with a scan rate of $25 \mathrm{kHz}(v=0.03)$, or a spectral-encoding scanner [9] with a rate of $50 \mathrm{kHz}$ $(v=0.06)$. These values are still an order of magnitude smaller than the limit $(v \sim 0.3)$ beyond which the resolution becomes speed-dependent, and thus the resolution is deteriorated. Therefore, there is apparently still room to push the laser scanning speed for LSFM higher. In this regard, we note that some possible laser-scanning solutions could have the potential, although they have not been utilized for LSFM, for biological specimens. For example, an AOD with a $100 \mathrm{kHz}$ scan rate $(v \sim 0.1)$ has been employed for nonfluorescent imaging [30]. In addition, the swept-source originally developed for optical coherence tomography (OCT) and spectrallyencoded imaging in a longer near-infrared (NIR) range

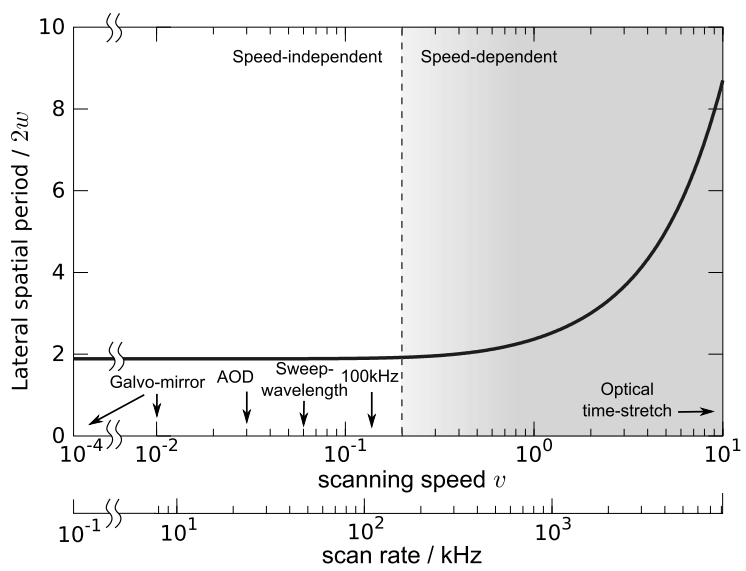

Fig. 5. Laser-scanning-speed-dependent resolution in LSFM. The scan speeds of the major laser scanning techniques are also labelled on the plot. Beyond $v \sim 0.2$, the resolution becomes speed-dependent (i.e., lifetime-limited). In contrast, the resolution is independent of the scan speed when $v \ll 0.2$ 
Table 1. Rayleigh Resolution of Selected Beam Scanning Mechanisms for LSFM

\begin{tabular}{|c|c|c|c|}
\hline Mechanism & $\begin{array}{l}\text { Sweep Rate } \\
(f / \mathrm{Hz})\end{array}$ & $\begin{array}{l}\text { Speed }^{a} \\
\quad(v)\end{array}$ & $\begin{array}{c}\text { Resolution }^{b} \\
\left(\pi / k_{\text {Ray }}\right)\end{array}$ \\
\hline $\begin{array}{l}\text { Galvanometric mirror, } \\
\text { nonresonant [5] }\end{array}$ & 500 & 0.0006 & $1.89 \times$ \\
\hline $\begin{array}{l}\text { Galvanometric mirror, } \\
\text { resonant }[5]\end{array}$ & 7800 & 0.010 & $1.89 \times$ \\
\hline $\mathrm{AOD}[\underline{8}]$ & $25 \mathrm{k}$ & 0.031 & $1.89 \times$ \\
\hline Swept-wavelength laser ${ }^{c}$ [9] & $50 \mathrm{k}$ & 0.062 & $1.89 \times$ \\
\hline KiloHertz laser scanner ${ }^{d}$ & $100 \mathrm{k}$ & 0.123 & $1.90 \times$ \\
\hline Optical time-stretch ${ }^{e}[39]$ & $10 \mathrm{M}$ & 12.3 & $10.46 \times$ \\
\hline
\end{tabular}

${ }^{a}$ Assuming $150 \mu \mathrm{m}$ full-sweep displacement or, equivalently, the field-of-view. This corresponds to the case using a typical high magnification OL.

${ }^{b}$ Lateral spatial period $\left(2 \pi / k_{\text {Ray }}\right)$ is normalized with respect to illumination beam waist diameter $2 w$ for clarity.

${ }^{c}$ Spectral-encoding scanner based on swept-wavelength laser.

${ }^{d}$ Examples are AOD [37], rotating polyonal mirrors [3], and spectral-encoding scanner [38].

${ }^{e}$ Not yet applied to LSFM.

( $\sim 800-1500 \mathrm{~nm})$, can now achieve a swept-rate of $>100 \mathrm{kHz}$ [31-34]. If this swept-source can be realized in the visible range, a spectral-encoded laser-scanning solution at multihundreds $\mathrm{kHz}$ could also be a viable solution to scale up the speed of LSFM to the limit.

Notably, the speed of laser-scanning based on spectralencoding can be further increased dramatically by optical time-stretch $[10,11,13,14]$. This is essentially a technique to map the spectrum of an ultrashort laser pulse ( $\mathrm{fs}-\mathrm{ps})$ into time via group velocity dispersion. This results in a wavelength-swept (chirped) temporal waveform with a scan rate as high as $\sim 1-10 \mathrm{MHz}$, which is governed by the repetition rate of the laser source. This corresponds to the scanning speed of $v \sim 1$ to $v \sim 10$-an interesting regime, where the resolution is dependent on the laser scanning speed, i.e., lifetimelimited resolution. We note that it might be possible to retrieve the diffraction-limited resolution in such a lifetime-limited regime by deconvolution techniques, provided that the fluorescence lifetime $\tau$ is known. It has been demonstrated that the lifetime can be estimated from the real-time measurements, even if the fluorescence decay is incomplete [35,36]. While such approach was taken in fluorescence lifetime imaging microscopy (FLIM), it could open up new possibility for image reconstruction in LSFM, if the scanning speed could be scaled up to $100 \mathrm{kHz}-1 \mathrm{MHz}$. Going beyond the scan rate of $v=10$ could severely worsen the resolution by the lifetime decay and, thus, seems not to be practical. For instance, a $10 \mathrm{MHz}$ time-stretch system $(v \sim 12)$ results in a resolution $\sim 10$ times worse than the diffraction-limited resolution. Table 1 summarizes the comparison among all aforementioned laser scanning techniques.

\section{B. Relationship between Spatial Resolution and Noise Level}

We further investigate the impact of noise on the resolution of LSFM over a wide range of scanning speeds $\left(v=10^{-4}\right.$ to $v=10$ ) by considering the speed-dependent detectability limit of the system (Fig. 6). Assuming unsaturated excitation (i.e., $0.1 \%, 1 \%$, and $10 \%$ of the saturation level), as well as shot-noise-limited condition, the resolution governed by detectability limit (red curve) is, in general, smaller than that determined by the Rayleigh criterion (blue dashed curve) in the low-speed regime $\left(v<10^{-2}\right)$. This corresponds to the case of Fig. 2(a). Therefore, in a low-speed LSFM system, the photon shot-noise does not have significant contribution to the actual resolution, which is mostly diffraction-limited.

As the scanning speed is scaled up, the detectability limit becomes comparable with, and even surpasses the $25 \%$ contrast level [i.e. Fig. 2(b)]. The resolution is now noise-limited, unless resorting to a higher excitation power. For instance, it is required to increase the excitation flux to at least $10 \%$ of the saturation level $\left(\bar{Q}_{\text {sat }}\right)$ to ensure the diffraction-limited resolution in the high-speed range of $v=0.01$ to $v=0.1$ (i.e., a scan rate of $\sim 10 \mathrm{kHz}-100 \mathrm{kHz}$ ) (Fig. 6). The SNR (=1/D), meanwhile, is also maintained at a reasonable level of $\sim 3 \%$ (Table 2). We note that this excitation flux level is practical in most of the biological imaging by LSFM [ $\underline{4}$ ].

Merely increasing the excitation power does not guarantee ultrafast LSFM. On an equal footing, a PD with high speed (bandwidth) and high sensitivity is also crucial. From Eqs. (18) and (19), we could estimate the required Nyquist sampling time $\bar{T}$ of photodetection under different speed regimes (see Table 2). In a low-speed system, e.g., using nonresonant scanning mirrors, the sampling time can be relaxed to $\sim 2,000 \times$ fluorescence lifetime. Considering the typical fluorescence lifetime of the fluorophores of $\sim 1-100 \mathrm{~ns}$, PDs with the time response $\sim 1 \mu \mathrm{s}-10 \mathrm{~ms}$, which are widely available, are adequate for commercial LSFM running in the low-speed regime.

In contrast, if the ultrafast LSFM is to be explored in the speed range of $v \sim 0.1$ to $v \sim 1$, one should employ high-speed detection with the required sampling time to be $\sim 10$ times the lifetime (Table 2). This implies that PDs with $>10 \mathrm{MHz}$ bandwidth (or nanoseconds temporal response time) are essential for such purpose. The current state-of-the-art PDs, including photomultiplier tubes (PMTs), avalanche photodiodes (APDs), and hybrid PDs (HPDs), could meet such a speed criterion with high detection sensitivity. Hence, they are the critical elements along with the ultrafast LSFM development.

Further pushing the speed $v \gg 1$ (scan rate $\gg 1 \mathrm{MHz}$ ) results in severe resolution degradation, not only because

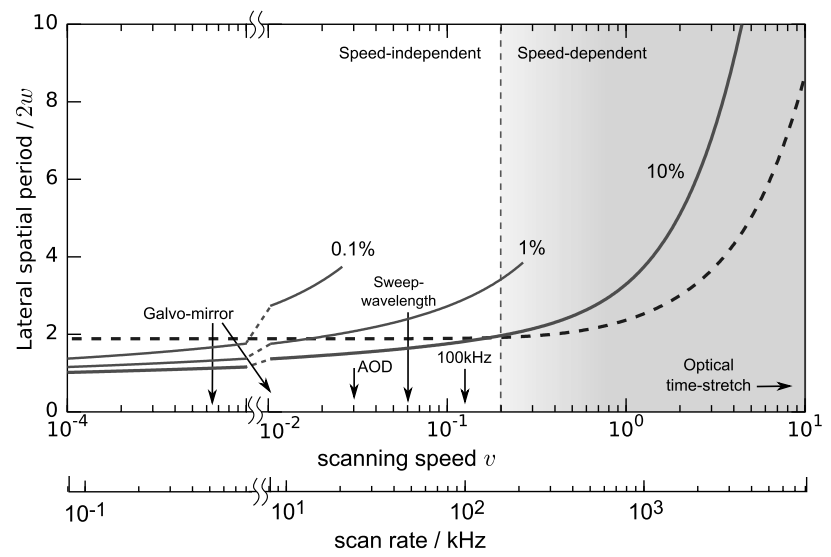

Fig. 6. Impact of noise on scanning-speed-dependent resolution of LSFM. Three excitation powers are chosen (red solid lines): 10\%, $1 \%$, and $0.1 \%$ of the saturation level. The three curves are obtained by evaluating the minimally resolvable feature size (in a unit of $2 w$ ), defined by detectability limit $D$, i.e., from the condition of Eq. (19). The actual resolution of the system is determined by the larger value of noise-limited resolution at a given excitation (red lines) and the resolution defined by the Rayleigh criterion (blue dashed line). 
Table 2. Required Nyquist-Sampling Interval and Detectability Limit of LSFM based on Selected Laser Scanning Mechanisms ${ }^{a}$

\begin{tabular}{|c|c|c|}
\hline Mechanism & $\begin{array}{l}\text { Sampling Interval }{ }^{b} \\
(T / \tau)\end{array}$ & $\begin{array}{l}\text { Detection Limit } \\
(D / \%)\end{array}$ \\
\hline $\begin{array}{l}\text { Galvanometric mirror, } \\
\text { nonresonant [5] }\end{array}$ & 1827. & 2.54 \\
\hline $\begin{array}{l}\text { Galvanometric mirror, } \\
\text { resonant [5] }\end{array}$ & 143. & 2.81 \\
\hline $\mathrm{AOD}[\underline{8}]$ & 49.8 & 2.97 \\
\hline Swept-wavelength laser ${ }^{c}[9]$ & 27.0 & 3.09 \\
\hline $100 \mathrm{kHz}$ laser scanner ${ }^{d}$ & 14.8 & 3.24 \\
\hline Optical time-stretch ${ }^{e}[11]$ & 2.2 & 12.57 \\
\hline
\end{tabular}

${ }^{a}$ Illumination power $\bar{Q}_{\mathrm{ex}}$ is assumed to be at $10 \%$ of saturation level $\bar{Q}_{\mathrm{sat}}$. ${ }^{b}$ Sampling interval $T=\pi /\left(v k_{\text {Det }}\right)$ is normalized with respect to lifetime $\tau$.

${ }^{c}$ Spectral-encoding scanner based on swept-wavelength laser.

${ }^{d}$ Examples are AOD [37], rotating polyonal mirrors [3], and spectral-encoding scanner [38].

${ }^{e}$ Not yet applied to LSFM.

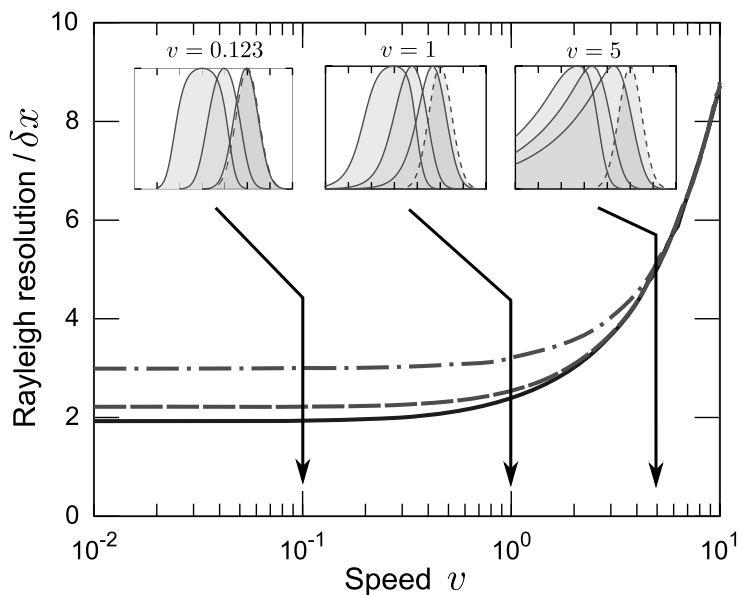

Fig. 7. Laser-scanning-speed-dependent resolution at three different excitation flux levels: $0.1 \times$ (solid line), $1 \times$ (dashed line), and $10 \times$ (dashed-dotted line) saturation level. The three insets show the saturation-dependent emission profiles upon Gaussian beam excitation (dotted blue) at the speed of $v=0.01, v=1$, and $v=5$. The emission profiles at the three excitation fluxes are shifted relative to each other by $2 w$ for clarity. From right to left: $0.1 \times, 1 \times$, and $10 \times$ saturation level $\bar{Q}_{\text {sat }}$. of lifetime decay, as mentioned in Section 4.A, but also because of the relationship between the Nyquist-limited sampling time of photodetection and the shot-noise-limited detectability limit (or SNR), which hinders high resolution at ultrafast scan speed [see Eq. (19)]. The sampling time (and thus the required photodetection response time) cannot always be scaled down with the increasing scan rate, especially when $v \gg 1$. This is because the response time should be bounded by a minimum time within which the shot-noiselimited SNR is still sufficient to provide distinguishable contrast, as determined by the detectability limit [Eq. (19)]. This noise-limited resolution is always worse than the diffractionlimited value. For an example, LSFM with a scan rate of $10 \mathrm{MHz}$ requires a sampling interval $\sim 3$ times of the lifetime, which gives rise to a detectability limit of $\sim 10 \%$. This leads to a resolution of 5 times of the diffraction-limited value (See Table $\underline{2}$ and Fig. $\underline{6}$ ).

\section{Impact of Fluorescence Saturation on Resolution}

It is clear from Fig. $\underline{6}$ that increasing the excitation power enhances the SNR, thus lowering the detectability limit and improving the resolution, if the system is noise-limited. When the power goes beyond the saturation level, i.e., the detectability limit could be brought below the 25\%-contrast level, even in the high speed regime. In this case, the resolution can be primarily determined by the Rayleigh criterion-either being diffraction-limited (at low speed, $v<1$ ) or lifetime-limited (at high speed, $v>1$ ). While it is well-known that saturation could modify the resolution in conventional LSFM $[28,40,41]$, we observe that this effect has a diminishing impact on the resolution as the scanning speed increases beyond $v>1$, i.e., lifetime-limited regime (Fig. 7). As expected, the diffraction-limited resolution (at $v=0.0 \overline{1}$ ) is degraded by saturation. It can be visualized by the broadened and flat-top-like emission profile at $10 \times$ saturation level (left-most inset of Fig. 7). The broadening effect is less obvious when the speed is increased up to $v=5$, at which the profile predominantly exhibits the long-tail decay and signifying the lifetime-limited resolution.

We finally perform numerical simulation of LSFM of a test pattern (checkerboard) at different scanning-speed regimes and excitation fluxes (Fig. ). The parameters employed in

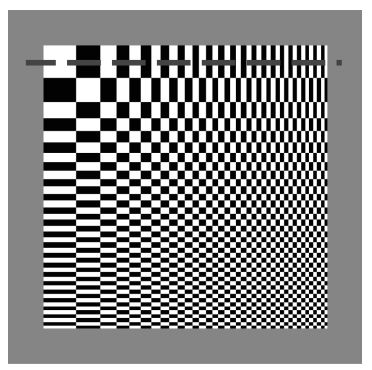

(a)

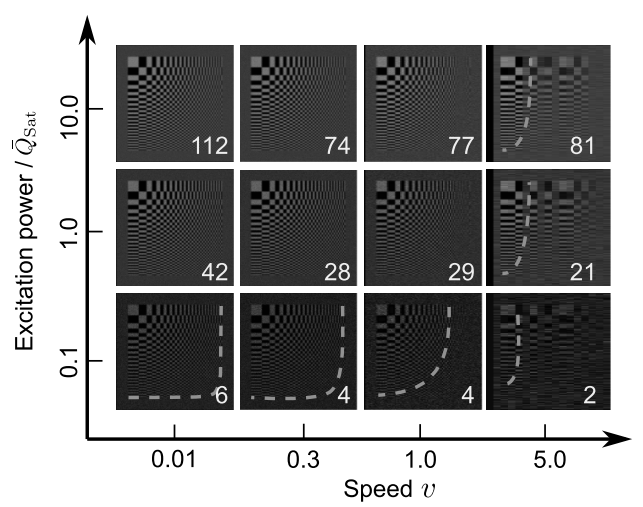

(b)

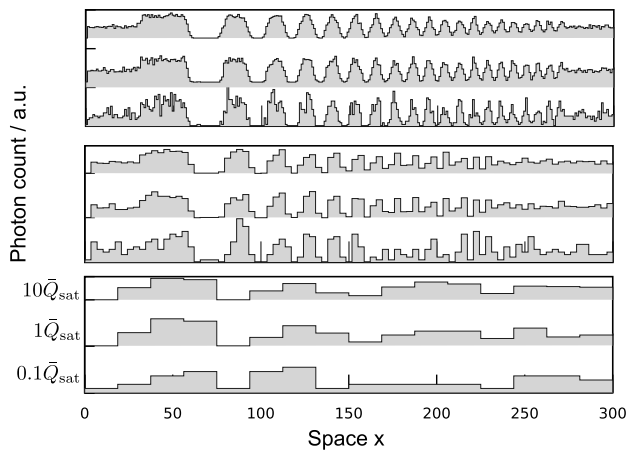

(c)

Fig. 8. (a) Test target pattern (with $150 \mu \mathrm{m}$ field-of-view) for numerical simulation of LSFM. The size of stripe pattern ranges from 2 to $20 \mu \mathrm{m}$. (b) Simulated image at different laser-scanning speeds (along the $x$-direction) and excitation flux levels $\left(\bar{Q}_{\text {ex }} / \bar{Q}_{\text {sat }}\right)$. The linear scan is along the $x$ direction. The average detected photon count per pixel is shown in each image. The dotted lines, which roughly indicate the positions of the minimally resolvable strips, are drawn for visual aid. (c) Corresponding line scan profiles at $v=0.01$, $v=1$, and $v=5$ along the dotted line illustrated in (a). 
Table 3. Numerical Values used in the Simulation

\begin{tabular}{|c|c|c|c|}
\hline Symbol & Value & Units & Description \\
\hline \multicolumn{4}{|c|}{ Illumination source: } \\
\hline$w$ & 0.5 & $\mu \mathrm{m}$ & Excitation beam waist radius \\
\hline$\Delta A$ & 1 & $\mu \mathrm{m}^{2}$ & Effective excitation cross-section area ${ }^{a}$ \\
\hline \multicolumn{4}{|c|}{ Fluorescence dye: } \\
\hline$\lambda_{\mathrm{ex}}$ & 494 & $\mathrm{~nm}$ & Peak excitation wavelength ${ }^{b}$ \\
\hline$\tau$ & 4.1 & ns & Effective fluorescence lifetime ${ }^{a}$ \\
\hline$a$ & $10^{-20}$ & $\mathrm{~m}^{2}$ & Effective absorption cross-section area \\
\hline$\eta_{q}$ & 0.9 & - & Fluorescence quantum efficiency \\
\hline $\bar{N}_{\text {tot }}^{2}$ & $0.2 \times 10^{-3}$ & $\mathrm{~mol} / \mathrm{m}^{3}$ & Average fluorescence concentration ${ }^{a}$ \\
\hline \multicolumn{4}{|c|}{ Optical system: } \\
\hline$\eta_{\text {obj }}$ & $10 \%$ & - & Collection efficiency of OL \\
\hline
\end{tabular}

the simulation are listed in Table $\underline{3}$ and represent the typical experimental conditions for biological imaging. Several key observations from this simulation include: (1) Both the resolution and the emission flux are compromised at higher speed. This is particularly obvious for $v>1$ that the image has to be pixelated to attain reasonable SNR (as discussed in Section 4.B); (2) The impact of the saturation on resolution becomes less significant for speed $v>1$; (3) It is plausible to push the scanning speed close to $v \sim 1$ while maintaining reasonably good resolution [with slight contamination by noise (Fig. 7)] and reasonable SNR, particularly in the saturation regime.

Based on Fig. 8, we could roughly estimate the key specifications of the $\mathrm{PD}$ required to operate in such an unexplored high-speed regime $(v=1)$. As the pixel sampling time for $v=1$ is $\sim 4 \mathrm{~ns}$, a conservative estimate of the PD's temporal response should be around or less than $1 \mathrm{~ns}$. Otherwise, its bandwidth should be on the order of GHz. Given that the average photon per pixel for $v=1$ is $\sim 2-81$, the noise-equivalent power (NEP) of the $1 \mathrm{GHz}$ PD should be on $\sim 10^{-14}$ $10^{-13} \mathrm{~W} / \mathrm{Hz}^{1 / 2}$ or less, assuming the emission wavelength of fluorescein $(494 \mathrm{~nm})$. While such specifications approach the limit of the current state-of-the-art sensitive PDs, e.g., PMTs, APDs, and HPDs, continuing advance in photodetection technologies could hold promise to bring the best combination of high-speed and high-sensitivity detection in the near future. This, together with the novel techniques of ultrafast laser scanning, could, therefore, open up a new speed paradigm in LSFM.

\section{CONCLUSION}

We present a theoretical analysis of LSFM in an underexplored high-speed laser-scanning regime, particularly where the fluorescence lifetime impacts image resolution. Based on our model that links the scanning speed, resolution, fluorescence saturation, and intrinsic photon shot-noise, we quantified the CTFs, detectability limit, and revisited the resolution evaluation of LSFM across a wide spectrum of laser-scanning speeds. At low scanning speeds, diffractionlimited resolution can easily be achieved without significant noise contamination. This corresponds to the classical laser scanning technology based on galvanometric mirrors, with a scan rate of no more than $\sim 10 \mathrm{kHz}$. If the speed is scaled up to $>100 \mathrm{kHz}$, which is made possible by AOD, or emerging techniques based on spectral-encoding, the resolution would likely worsen by the presence of noise, due to shorter dwell time at higher speed. With sufficiently high excitation flux in the ultrahigh speed regime, the resolution could be lifetimelimited, as the fluorescence decay creates significant cross talk with the neighboring excitation spots and, thus, degrades the resolution. In this case, the diffraction-limited resolution could possibly be retrieved using proper deconvolution techniques with the knowledge of the lifetime, thus representing a new approach for image reconstruction in ultrafast LSFM. We also found that the saturation effect does not play an important role in determining the actual resolution in the high-speed regime. Based on the model, we identified that there still exists a gap between the speed achieved by the typical laserscanning technologies and the speed limit beyond which the lifetime or/and the noise greatly degrade the resolution, i.e., the regime of $v=0.1$ to $v=1$. This corresponds to a scan rate of $\sim 100 \mathrm{kHz}-1 \mathrm{MHz}$, which is rather under-explored. We note that both AOD and spectral-encoding-based laserscanning could possibly be the viable candidates to achieve such scanning speeds. Together with the innovation of highspeed and sensitive PDs, ultrafast LSFM with a scan rate of sub-MHz to $\mathrm{MHz}$ should not be far from reach. We anticipate that the present study provides new insight to developing ultrafast LSFM, which could benefit high-speed dynamical studies of biological systems, as well as high-throughput screening applications.

\section{APPENDIX A: DERIVATION OF EQUATIONS (11) AND (12)}

By taking Fourier transform of Eqs. (9) and (10) with respect to time $t$, we have

$$
\begin{array}{r}
l \omega \tilde{p}_{1}(x ; \omega)=-\tilde{p}_{1}(x ; \omega)+\eta_{\mathrm{abs}} \bar{Q}_{\mathrm{ex}} \\
\times \frac{\sqrt{2 \pi}}{v} \exp \left(-\frac{\omega^{2}}{2 v^{2}}\right) \exp \left(\frac{\imath x \omega}{v}\right), \\
\tilde{Q}_{\mathrm{em}}(\omega)=\eta_{\mathrm{obj}} \eta_{q} \int_{-\infty}^{+\infty} N_{\mathrm{tot}}(x) \tilde{p}_{1}(x ; \omega) \mathrm{d} x w \Delta A .
\end{array}
$$

If we define lateral spatial frequency $k=\omega / v$, then the above equations become more elegant:

$$
\imath v k \tilde{p}_{1}=-\tilde{p}_{1}+\eta_{\mathrm{abs}} \bar{Q}_{\mathrm{ex}} \times \frac{\sqrt{2 \pi}}{v} \exp \left(-\frac{1}{2} k^{2}\right) \exp (\iota x k)
$$

$$
\tilde{Q}_{\mathrm{em}}(\omega)=\eta_{\mathrm{obj}} \eta_{q} \int_{-\infty}^{+\infty} N_{\mathrm{tot}}(x) \tilde{p}_{1} \mathrm{~d} x w \Delta A
$$

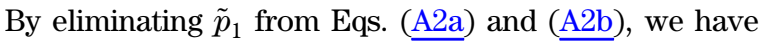

$$
\begin{aligned}
\tilde{Q}_{\mathrm{em}}(\omega)= & \eta_{\mathrm{obj}} \eta_{q} \eta_{\mathrm{abs}} \frac{\exp \left(-k^{2} / 2\right)}{1+\imath v k} \times \frac{\sqrt{2 \pi}}{v} \int_{-\infty}^{+\infty} N_{\mathrm{tot}}(x) \\
& \times \exp (\iota x k) \mathrm{d} x w \Delta A \times \bar{Q}_{\mathrm{ex}} .
\end{aligned}
$$

As the integrand is a spatial frequency spectrum of $N_{\text {tot }}(x)$, defined as $\tilde{N}_{\text {tot }}(k)$, Eq. (A3) can be written as 


$$
\begin{aligned}
\frac{\tilde{Q}_{\mathrm{em}}(\omega)}{\bar{Q}_{\mathrm{ex}}} & =\eta_{\mathrm{obj}} \eta_{q} \eta_{\mathrm{abs}} \frac{\exp \left(-k^{2} / 2\right)}{1+\imath v k} \times \frac{\sqrt{2 \pi}}{v} \tilde{N}_{\mathrm{tot}}(k) w \Delta A \Rightarrow H(k) \\
& =\frac{v \tilde{Q}_{\mathrm{em}}(\omega) / \bar{Q}_{\mathrm{ex}}}{\tilde{N}_{\mathrm{tot}}(k)}=\eta_{\mathrm{obj}} \eta_{q} \eta_{\mathrm{abs}} \frac{\exp \left(-k^{2} / 2\right)}{1+\imath v k} \sqrt{2 \pi} w \Delta A .
\end{aligned}
$$

In the case of uniform concentration, $\tilde{N}_{\text {tot }}(k)$ and $\tilde{Q}_{\mathrm{em}}(\omega)$ are Dirac delta functions. The transfer function in Eq. (A4) then consists of only efficiency factors, i.e.,

$$
\begin{aligned}
& \frac{v \bar{Q}_{\mathrm{em}} \delta(\omega) / \bar{Q}_{\mathrm{ex}}}{\bar{N}_{\mathrm{tot}} \delta(k)}=\eta_{\mathrm{obj}} \eta_{q} \eta_{\mathrm{abs}} \times \sqrt{2 \pi} w \Delta A \\
& \quad \Rightarrow \gamma=\frac{\bar{Q}_{\mathrm{em}}}{\bar{Q}_{\mathrm{ex}}}=\eta_{\mathrm{obj}} \eta_{q} \eta_{\mathrm{abs}} \times \bar{N}_{\mathrm{tot}} \times \sqrt{2 \pi} w \Delta A .
\end{aligned}
$$

This is the same expression as Eq. (11). Note that the speed factor on the left hand side vanishes because of the relative strength of the Dirac delta function, i.e., $\delta(k)=v \delta(\omega)$. Since we are interested in the absolute response, we normalize the transfer function:

$$
\operatorname{CTF}(k)=\frac{|H(k)|}{\gamma}=\frac{\exp \left(-k^{2} / 2\right)}{\sqrt{1+v^{2} k^{2}}} .
$$

\section{ACKNOWLEDGMENTS}

The author would like to thank Dr. Ray Xu for discussions and reading the manuscript. This work is partially supported by grants from the Research Grants Council of Hong Kong Special Administrative Region, China (HKU 717510E, HKU 717911E, HKU 720112E, and HKU 713811E), and the University Development Fund of HKU.

\section{REFERENCES}

1. J. Pawley, "Fundamental limits in confocal microscopy," in Handbook of Biological Confocal Microscopy (Springer, 2006), pp. 20-42.

2. H. R. Petty, "High speed microscopy in biomedical research," Opt. Photon. News 15(1), 40-45 (2004).

3. K. H. Kim, C. Buehler, and P. T. So, "High-speed, two-photon scanning microscope," Appl. Opt. 38, 6004-6009 (1999).

4. T. F. Holekamp, D. Turaga, and T. E. Holy, "Fast threedimensional fluorescence imaging of activity in neural populations by objective-coupled planar illumination microscopy," Neuron 57, 661-672 (2008).

5. J. M. Larson, S. A. Schwartz, and M. W. Davidson, "Resonant scanning in laser confocal microscopy," Tech. Rep., (Nikon Microscopy, 2000).

6. S. Choi, P. Kim, R. Boutilier, M. Kim, Y. Lee, and H. Lee, "Development of a high speed laser scanning confocal microscope with an acquisition rate up to 200 frames per second," Opt. Express 21, 23611-23618 (2013).

7. J. Xu and R. Stroud, Acousto-Optic Devices: Principles, Design, and Applications (Wiley, 1992).

8. V. Bansal, S. Patel, and P. Saggau, A High-Speed Confocal Laser-Scanning Microscope Based on Acousto-Optic Deflectors and a Digital Micromirror Device (Institute of Electrical and Electronics Engineers, 2003), pp. 2124-2127.

9. M. Strupler, E. D. Montigny, D. Morneau, and C. Boudoux, "Rapid spectrally encoded fluorescence imaging using a wavelength-swept source," Opt. Lett. 35, 1737-1739 (2010).

10. K. Goda and B. Jalali, "Dispersive Fourier transformation for fast continuous single-shot measurements," Nat. Photonics 7, 102-112 (2013).
11. K. Goda, K. K. Tsia, and B. Jalali, "Serial time-encoded amplified imaging for real-time observation of fast dynamic phenomena," Nature 458, 1145-1149 (2009).

12. K. Goda, A. Ayazi, D. R. Gossett, J. Sadasivam, C. K. Lonappan, E. Sollier, A. M. Fard, S. C. Hur, J. Adam, C. Murray, C. Wang, N. Brackbill, D. Di Carlo, and B. Jalali, "High-throughput singlemicroparticle imaging flow analyzer," Proc. Natl. Acad. Sci USA 109, 11630-11635 (2012).

13. Y. Qiu, J. Xu, K. K. Y. Wong, and K. K. Tsia, "Exploiting few mode-fibers for optical time-stretch confocal microscopy in the short near-infrared window," Opt. Express 20, 24115-24123 (2012).

14. T. T. Wong, A. K. Lau, K. K. Wong, and K. K. Tsia, "Optical timestretch confocal microscopy at $1 \mu \mathrm{m}$," Opt. Lett. 37, 3330-3332 (2012).

15. C. Zhang, Y. Qiu, R. Zhu, K. K. Wong, and K. K. Tsia, "Serial time-encoded amplified microscopy (STEAM) based on a stabilized picosecond supercontinuum source," Opt. Express 19, 15810-15816 (2011).

16. A. Mahjoubfar, C. Chen, K. R. Niazi, S. Rabizadeh, and B. Jalali, "Label-free high-throughput cell screening in flow," Biomed. Opt. Express 4, 1618-1625 (2013).

17. P. I. Bastiaens and A. Squire, "Fluorescence lifetime imaging microscopy: spatial resolution of biochemical processes in the cell," Trends Cell Biol. 9, 48-52 (1999).

18. J. C. Waters, "Accuracy and precision in quantitative fluorescence microscopy," J. Cell Biol. 185, 1135-1148 (2009).

19. K. Fujita, M. Kobayashi, S. Kawano, M. Yamanaka, and S. Kawata, "High-resolution confocal microscopy by saturated excitation of fluorescence," Phys. Rev. Lett. 99, 228105 (2007).

20. E. Stelzer, "Contrast, resolution, pixelation, dynamic range, and signal-to-noise ratio: fundamental limits to resolution in fluorescence light microscopy,” J. Microsc. 189, 15-24 (1998).

21. O. Nakamura and S. Kawata, "Three-dimensional transferfunction analysis of the tomographic capability of a confocal fluorescence microscope,” J. Opt. Soc. Am. 7, 522-526 (1990).

22. H. R. Petty, "Fluorescence microscopy: established and emerging methods, experimental strategies, and applications in immunology," Microsc. Res. Tech. 70, 687-709 (2007)

23. J. Philip and K. Carlsson, "Theoretical investigation of the signalto-noise ratio in fluorescence lifetime imaging," J. Opt. Soc. Am. 20, 368-379 (2003)

24. Q. Zhao, I. T. Young, and J. G. S. de Jong, "Photon budget analysis for fluorescence lifetime imaging microscopy," J. Biomed. Opt. 16, 086007 (2011).

25. D. Semwogerere and E. R. Weeks, Confocal Microscopy (Encyclopedia of Biomaterials and Biomedical Engineering, 2005).

26. E. Wang, C. M. Babbey, and K. W. Dunn, "Performance comparison between the high-speed Yokogawa spinning disc confocal system and single-point scanning confocal systems," J. Microsc 218, 148-159 (2005).

27. B. Zhang, J. Zerubia, and J.-C. Olivo-Marin, "Gaussian approximations of fluorescence microscope point-spread function models," Appl. Opt. 46, 1819-1829 (2007).

28. K. Visscher, G. J. Braeckmans, and T. D. Visser, "Fluorescence saturation in confocal microscopy," J. Microsc. 175, 162-165 (1994).

29. J. W. Daily, "Saturation effects in laser induced fluorescence spectroscopy," Appl. Opt. 16, 568-571 (1977).

30. K. Goda, A. Mahjoubfar, C. Wang, A. Fard, J. Adam, D. R Gossett, A. Ayazi, E. Sollier, O. Malik, E. Chen, Y. Liu, R. Brown, N. Sarkhosh, D. Di Carlo, and B. Jalali, "Hybrid dispersion laser scanner," Sci. Rep. 2, 1-8 (2012).

31. S. C. Schlachter, D. Kang, M. J. Gora, P. Vacas-Jacques, T. Wu, R. W. Carruth, E. J. Wilsterman, B. E. Bouma, K. Woods, and G. J. Tearney, "Spectrally encoded confocal microscopy of esophageal tissues at $100 \mathrm{kHz}$ line rate," Biomed. Opt. Express 4, 1636-1645 (2013).

32. R. Huber, D. C. Adler, V. J. Srinivasan, and J. G. Fujimoto, "Fourier domain mode locking at $1050 \mathrm{~nm}$ for ultra-high-speed optical coherence tomography of the human retina at 236,000 axial scans per second," Opt. Lett. 32, 2049-2051 (2007).

33. H.-C. Lee, J. J. Liu, Y. Sheikine, A. D. Aguirre, J. L. Connolly, and J. G. Fujimoto, "Ultrahigh speed spectral-domain optical coherence microscopy,” Biomed. Opt. Express 4, 1236-1254 (2013). 
34. W. Wieser, B. R. Biedermann, T. Klein, C. M. Eigenwillig, and R. Huber, "Multi-megahertz oct: high quality 3D imaging at 20 million a-scans and 4.5 GVoxels per second," Opt. Express 18, 14685-14704 (2010).

35. Y. Yuan, T. Papaioannou, and Q. Fang, "Single-shot acquisition of time-resolved fluorescence spectra using a multiple delay optical fiber bundle," Opt. Lett. 33, 791-793 (2008).

36. R. W. K. Leung, S.-C. A. Yeh, and Q. Fang, "Effects of incomplete decay in fluorescence lifetime estimation," Biomed. Opt. Express 2, 2517-2531 (2011).

37. R. Salom, Y. Kremer, S. Dieudonn, J.-F. Lger, O. Krichevsky, C. Wyart, D. Chatenay, and L. Bourdieu, "Ultrafast randomaccess scanning in two-photon microscopy using acoustooptic deflectors," J. Neurosci. Methods 154, 161-174 (2006).
38. M. W. Jenkins, D. C. Adler, M. Gargesha, R. Huber, F. Rothenberg, J. Belding, M. Watanabe, D. L. Wilson, J. G. Fujimoto, and A. M. Rollins, "Ultrahigh-speed optical coherence tomography imaging and visualization of the embryonic avian heart using a buffered Fourier-domain-mode locked laser," Opt. Express 15, 6251-6267 (2007).

39. K. K. Tsia, K. Goda, D. Capewell, and B. Jalali, "Performance of serial time-encoded amplified microscope," Opt. Express 18, 10016-10028 (2010).

40. G. C. Cianci, J. Wu, and K. M. Berland, "Saturation modified point spread functions in two-photon microscopy," Microsc. Res. Tech. 64, 135-141 (2004).

41. R. M. Doornbos, B. G. de Grooth, and J. Greve, "Experimental and model investigations of bleaching and saturation of fluorescence in flow cytometry," Cytometry 29, 204-214 (1997). 